\title{
An Efficient Navigation Method for Virtual Endoscopy Using Volume Ray Casting
}

\author{
Byeong-Seok Shin and Suk Hyun Lim \\ Inha University, Department of Computer Science and Engineering \\ 253 Yonghyeon-Dong, Nam-Gu, Inchon, 402-751, Korea \\ bsshinainha.ac.kr \\ q2011498@inhavision.inha.ac.kr
}

\begin{abstract}
In virtual endoscopy, it is important to devise the navigation method that enables us to control the position and orientation of virtual camera easily and intuitively without collision to organ wall. We propose an efficient navigation algorithm that calculates depth information while rendering a scene for current frame with volume ray casting method, then computes new viewing specification of camera for the next frame using the depth information. It can generate a camera path in real-time, allows us to control the camera with ease, and avoids collision with objects efficiently. In addition, it doesn't require preprocessing stage and extra storage for maintaining spatial data structures. Experimental result shows that it is possible to navigate through human colon when we apply our method to virtual colonoscopy.
\end{abstract}

\section{Introduction}

Optical endoscopy is a less-invasive diagnosis method. We can directly examine the pathologies of internal organs by putting endoscopy camera into human body. It offers the highest quality images than any other medical imaging methods. However it has some disadvantages of causing patients discomfort, limited range of exploration and serious side effect such as perforation, infection and hemorrhage.

Virtual endoscopy is regarded as a good alternative of optical endoscopy. After acquiring cross-sectional images of human abdomen with CT or MRI, we can reconstruct three dimensional volume models and provide visualizations of inner surface of the human organ, which has pipe-like shape such as colon, bronchi, and blood vessels. Since the virtual endoscopy is non-invasive examination, there is no discomfort and side effects. It is adequate for mass screening of pathological cases and training tool for doctors and medical students.

In order to implement virtual endoscopy based on volume rendering technique, we should devise a method that generates high quality perspective images within short time. However, it is more important to detect collision between virtual camera and organ wall in real-time, and let the camera smoothly move along the center-line of human cavity. Since the organs to be examined such as colon and bronchus have 
complex structures, it is difficult to move forward endoscopy camera and change its orientation even for experienced doctors. So, fast and accurate navigation is essential for efficient diagnosis using virtual endoscopy. Previous methods for navigation have some problems to demand a lot of computation cost in preprocessing step and it doesn't allow user to change the camera position and orientation during navigation, or to require extra storages for spatial data structures such as a distance map or a potential field.

In this paper, we propose an efficient algorithm that generates depth information while rendering a scene in current frame with volume ray casting, then determines camera orientation for the next frame using the information. Since the depth information can be estimated without additional cost during ray casting, it computes camera path in real-time. In addition, it doesn't require extra memory and preprocessing step.

Related works is summarized in the next section. In section 3, we present our algorithm in detail. Experimental results and remarks are shown in section 4. Lastly, we summarize and conclude our work.

\section{Related Work}

Volume ray casting is the most famous volume rendering method [1]. After firing a ray from each pixel on the view plane into volume space, it computes color and opacity on sample points along the ray using the corresponding voxel values and opacity transfer function. Then it determines final color for the pixel by blending them. Although it takes long time to make an image due to randomness in memory reference pattern, it produces high-quality images in comparison to the other methods. Also it can make perspective images such as endoscopic image. It is possible to improve its rendering speed using some acceleration technique such as template based rendering [2], shear-warp decomposition [3], and space-leaping [4].

In order to implement realistic virtual environment, several kinds of interactions between objects should be considered. Especially, collision detection is more important than any other components for visual realism [5]. We have to devise appropriate way to specify the surface of volumetric objects since they do not have explicitly defined surfaces unlike geometric objects. Usually we define object boundaries indirectly using the opacity transfer function, which assigns higher opacity values to voxels regarded as boundaries, and lower opacities to the others. This process is called classification. Several spatial data structure might be used to detect collision between an object and the indirectly specified surfaces.

Occupancy map has the same resolution as the target volume dataset and each cell of the map stores identifiers for objects that occupy the cell [6]. As the objects change its position, the values of each cell should be updated. If a cell has two or more identifiers, it is recognized as collision of those objects. This method requires large amount of storage as original volume dataset, and it should update its contents whenever an object change its position and size.

Three dimensional distance map can be used for collision detection and avoidance [7]. A distance map is a 3D spatial data structure that has the same resolution of its 
volume data and each point of the map has the distance to the nearest boundary voxel of an object. We can exploit the distance information generated in preprocessing time for collision detection without extra cost. The smaller the distance values the larger the collision probability and vice versa. Asymptotic complexity of collision detection is $O(1)$, since we have to refer the distance map only once. Due to the occupancy map, this method requires large amount of storage and considerably long preprocessing time.

Navigation method for virtual endoscopy can be classified into three categories; manual navigation [8], planned navigation [9] and guided navigation [10]. In manual navigation, we can directly control the virtual camera to observe anywhere we want to examine. However the user might feel discomfort since camera movement is entirely dependent on user's control, and collision with organ wall may occur if the user misleads the camera.

Planned navigation calculates entire navigation path in preprocessing time using several path generation algorithm, then moves through the camera along the precalculated path in navigation step. It can fly through the desired area without user intervention. However it requires a lot of computation cost in preprocessing step and it doesn't allow users to control the camera position and orientation intuitively.

Guided navigation is a physically-based method, which makes an spatial data structures such as potential field in preprocessing step and determines camera orientation by considering attractive force that directs from starting point to target point, repulsive force from organ surface, and user's input. It guarantees that a camera arrives to target point and move the camera to anywhere the user want to place intuitively without collision against organ wall. However it is very hard to implement and it demands a lot of cost to make and maintain the vector volume just like a potential field.

\section{Navigation Method Using Volume Ray Casting}

In order to implement efficient navigation, it have to satisfy the following requirements. (1) It should avoid collision between organ surface and virtual camera. (2) It should guarantee smooth movement of camera, and camera orientation should not change abruptly. (3) It should allow users to control the camera conveniently and intuitively. (4) It should not require preprocessing step, or minimize that if needed. (5) It should not require extra storages.

Guided navigation algorithms mentioned in the previous section is regarded as $o b$ ject-space method since they exploit overall information of entire volume dataset. Even though it guarantees accurate navigation, this is a reason why it takes a long preprocessing time. In virtual endoscopy, it requires information only for a small part of entire volume since field of view is restricted in cavities of a volume data set. Our navigation algorithm computes camera orientation using voxels visible in current viewing condition, thus we define it as image-space method. 
In rendering point of view, navigation is a kind of animation sequence that moves a camera along a smooth path. Here, we explain how to determine camera orientation for the next frame $f_{i+1}$, using the information obtained in rendering time for current frame $f_{i}$. We determine the direction of rotation using the largest depth value, and angle (magnitude) of rotation based on the smallest value. Figure 1 shows the procedure of our method. Rendering process is the same as in the conventional animation. Our method has another pipeline to specify the viewing condition of camera. It generates depth information while rendering and computes direction of rotation and rotation angle by using the depth information.

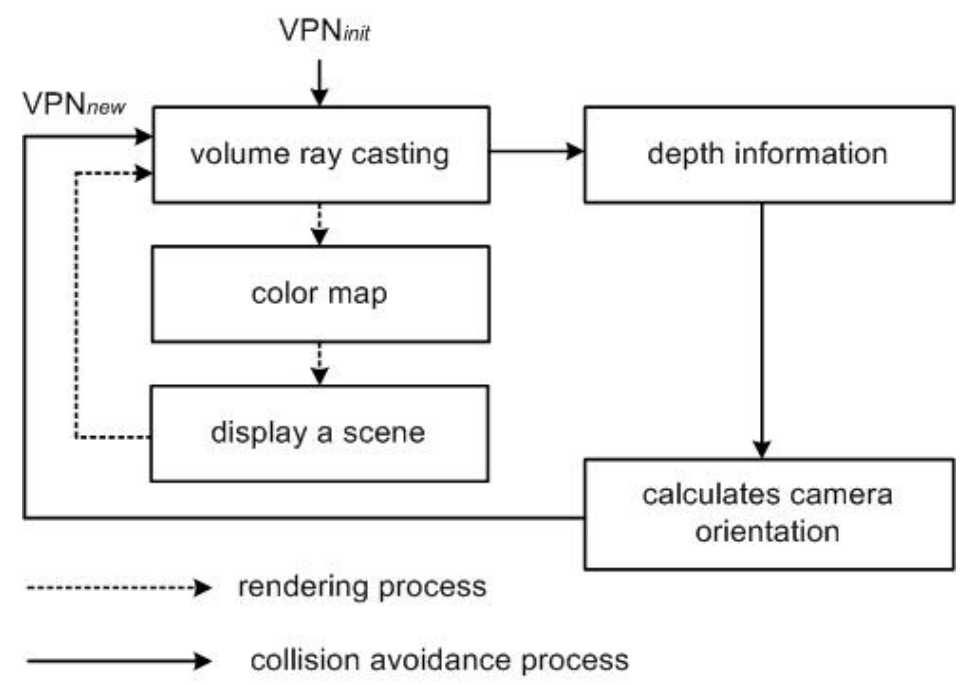

Fig. 1. A procedure for determining reliable navigation path

\subsection{Calculating Depth Information}

Volume ray casting fires a ray from each pixel on the view plane into volume space, then it computes color and opacity on sample points along the ray and determines final color for the pixel by blending them. Empty space (cavities) of volume data has low opacity. On the contrary organ wall has relatively high opacity value. While sampling voxel values along a ray, we can compute the distance from a pixel to the nearest non-transparent voxel using the number of samples and unit distance between two consecutive samples. Figure 2 depicts how to obtain distance value for each pixel. In our application, the distance can be regarded as the depth value from a camera to organ wall.

Actually, we need only the maximum and minimum depth value for the following steps. Let $d_{i, j}$ be a depth value of a pixel $p_{i, j}$, and $\mathbf{R}_{i, j}$ be the direction of ray for that pixel. Minimum and maximum depth value $d_{\min }$ and $d_{\max }$ can be defined as follows: 


$$
d_{\min }=\min \left(\bigcup_{i, j=0}^{M, N} d_{i, j}\right), d_{\max }=\max \left(\bigcup_{i, j=0}^{M, N} d_{i, j}\right) .
$$

where $M$ and $N$ are horizontal and vertical resolution of final image respectively. Also we store the direction of a ray fired from a pixel that has the maximum depth value as a form of unit vector, which is denoted as $\mathbf{R}_{\max }$.

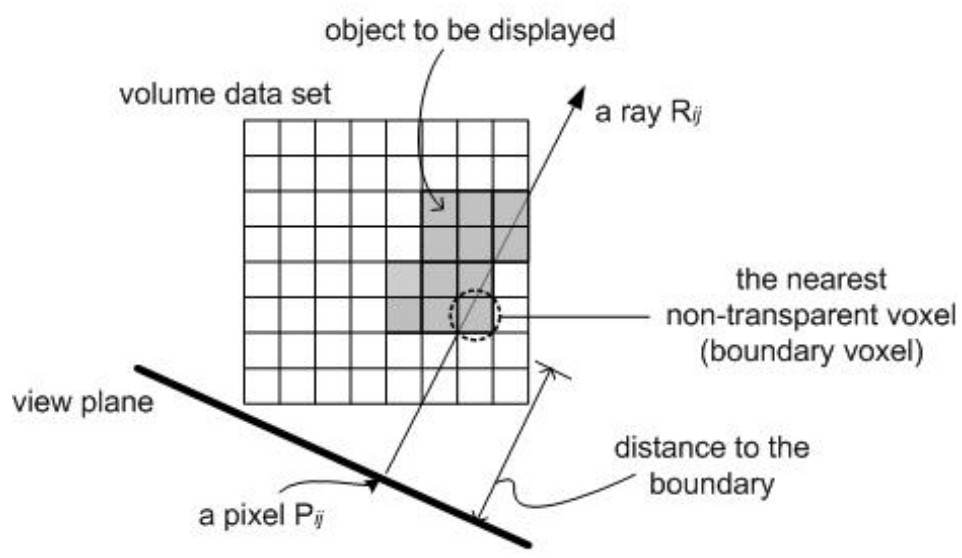

Fig. 2. Computing distance from a pixel to the nearest non-transparent voxel (is potentially regarded as organ boundary)

\subsection{Determining the Direction of Rotation of Virtual Camera}

Depth values are inverse proportional to the possibility of collision. Larger depth value means that obstacles are located far away from the current camera position. On the contrary, smaller the depth value, higher the collision possibility since the camera is near the organ wall. In this case, we have to rotate the camera rapidly to avoid collision. That is, we can minimize the collision possibility by altering the camera orientation to the direction of ray that has the maximum depth value.

We can define the direction of rotation $\mathbf{r}_{i}$ for the next frame $f_{i+1}$ can be defined as follows:

$$
\mathbf{r}_{i}=\mathbf{R}_{\max }-\mathbf{V P N _ { i }}
$$

where $\mathbf{R}_{\max }$ is a unit vector computed in the previous step, and $\mathbf{V P N}$ is also a unit vector that represents a view plane normal for current frame $f_{i}$. Figure 3 shows how to determine the direction of rotation using $\mathbf{R}_{\max }$. 


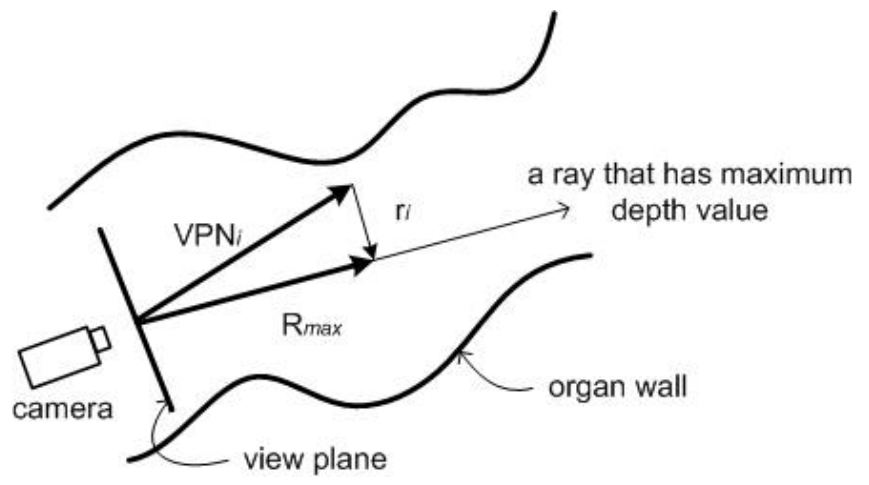

Fig. 3. Determination of the direction of rotation using the maximum distance value

\subsection{Calculating the Rotation Angle}

After determining the direction of rotation $\mathbf{r}_{i}$, we have to calculate rotation angle using minimum depth value $d_{\min }$. As we mentioned before, smaller $d_{\min }$ implies that a camera is located near the organ wall, so we should increase the rotation angle along $\mathbf{r}_{i}$. On the contrary, larger $d_{\min }$ decreases the angle.

It is simple but has a problem of instability. Since our method changes the camera orientation in every frame, unnecessary changes might occur in open area as shown in Figure 4. This causes camera trembling, so it cannot satisfy the requirement (2). To solve the instability problem, we define threshold depth value $d_{t h}$, then we do not alter camera orientation in sufficiently open area by considering the value. If $d_{\min }$ is larger than $d_{t h}$, it is regarded as in open area and we have to keep the camera orientation unchanged.

We can determine the rotation angle $\theta$ based on $d_{t h}$ and $d_{\min }$ as follows:

$$
\theta=\left\{\begin{array}{ll}
0 & \text { if } d_{\min }>d_{t h} \\
\arctan \left(\frac{d_{t h}-d_{\min }}{d_{t h}}\right) & \text { otherwise }
\end{array} .\right.
$$

Figure 5 shows how to determine the rotation angles according to the difference between $d_{t h}$ and $d_{\min }$.

In consequence, we can obtain the final camera orientation $\mathbf{V P N}_{i+1}$ for the text frame as in the follow equation:

$$
\mathbf{V P N}_{i+1}=\frac{\mathbf{r}_{i} \tan \theta+\mathbf{V P N}}{\left|\mathbf{r}_{i} \tan \theta+\mathbf{V P N}_{i}\right|}
$$




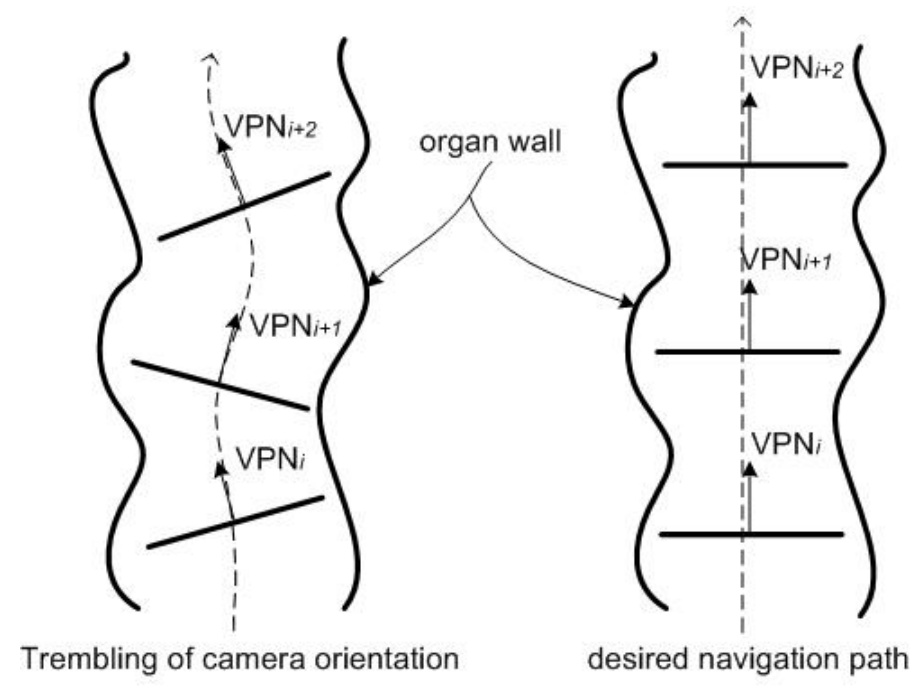

Fig. 4. An example of trembling of camera orientation in open area. It should be fixed along the desired navigation path.

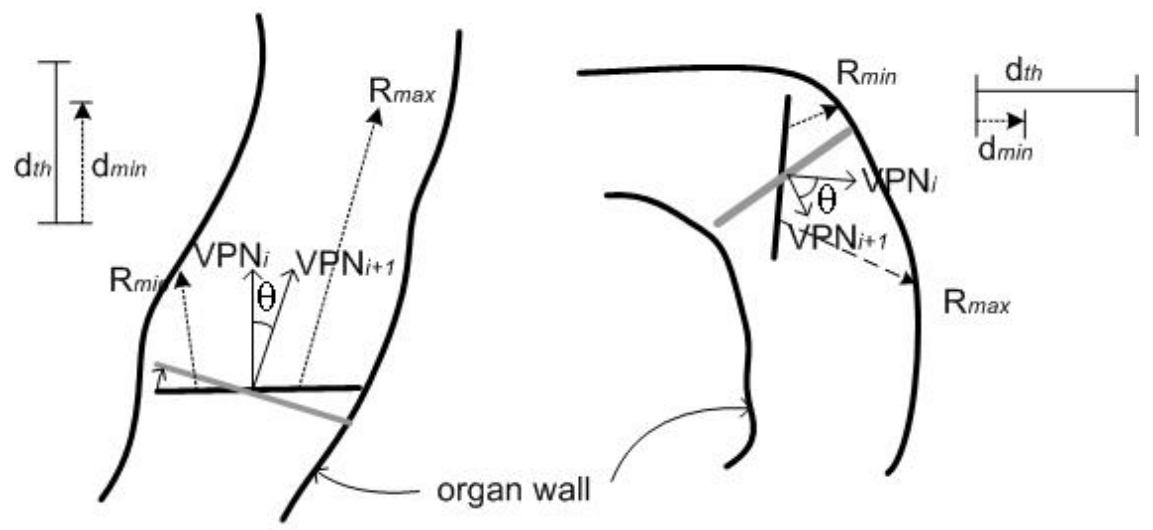

Fig. 5. Determination of the rotation angle using the minimum distance value

\section{Experimental Results}

In this section we will check whether our method offers reliable navigation through human colon with collision avoidance. Also we will compare the preprocessing and rendering time of a typical object-space navigation method based on distance transformation and our navigation algorithm. All of these methods are implemented on a 
PC which has Pentium IV 1.7GB CPU, 1GB main memory, and ATI RADEON 9000 graphics accelerator. The volume data used for the experiment is a clinical volume obtained by scanning a human abdomen with a spiral CT (computed tomography) with resolution $512 \times 512 \times 541$.

Figure 6 depicts the result of the method that does not perform collision detection and avoidance. In this case, a camera would bump against colon wall unless a user manually changes its direction. Figure 7 shows the example of using collision avoidance feature of our method in virtual colonoscopy. As you can see, the camera could move forward along the colon cavity automatically.

Table 1 shows the comparison of preprocessing time and rendering time of a method that does not perform any kind of collision avoidance (NONE), an objectspace guided navigation method using distance map (DIST) and our image space navigation method (OURS). While an object-space method spends long time for generating a distance map in preprocessing step, an image-space method doesn't need preprocessing time at all. In rendering time, the difference between NONE and OURS is very small (about $6.51 \%$ ), which implies that our method requires small amount of additional cost to calculate camera orientation during rendering. Also our navigation method is not so slow (only $4.91 \%$ ) even in case of comparing it with DIST.

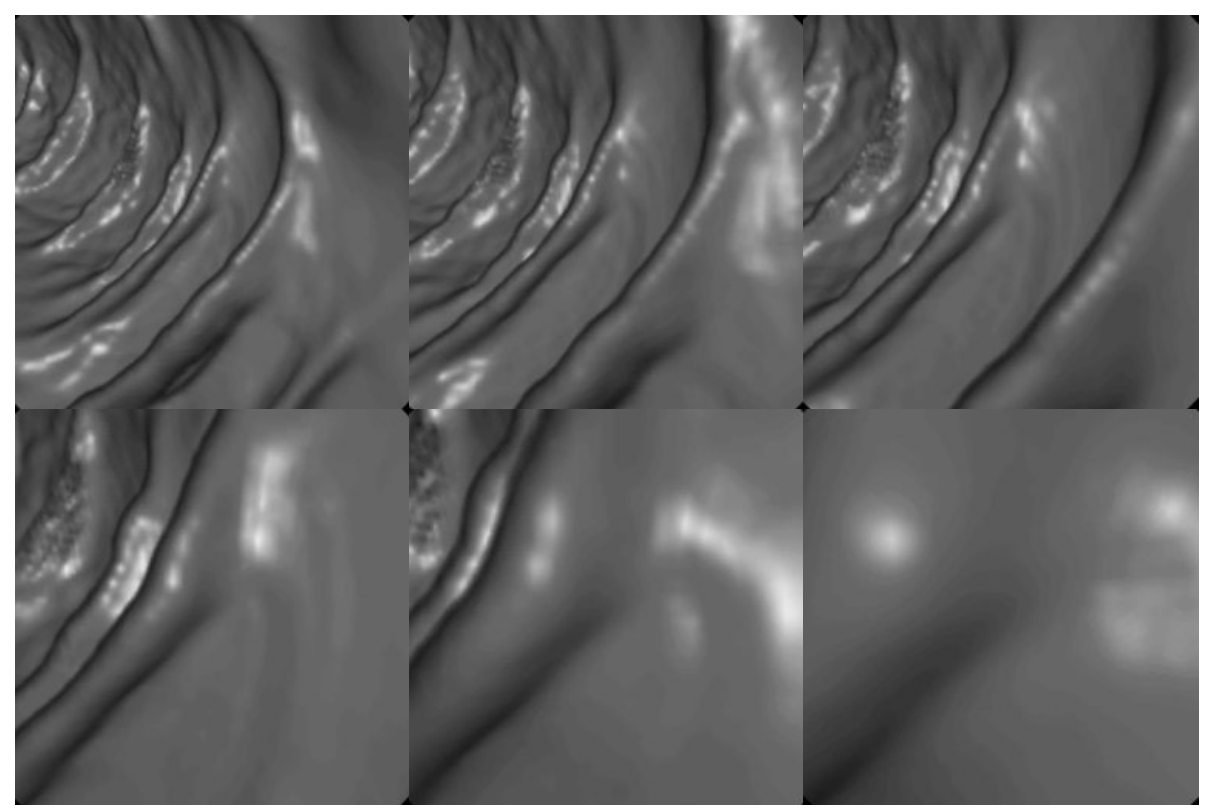

Fig. 6. This is an example of not using collision detection and avoidance feature. A camera would bump against colon wall unless a user manually changes its direction 


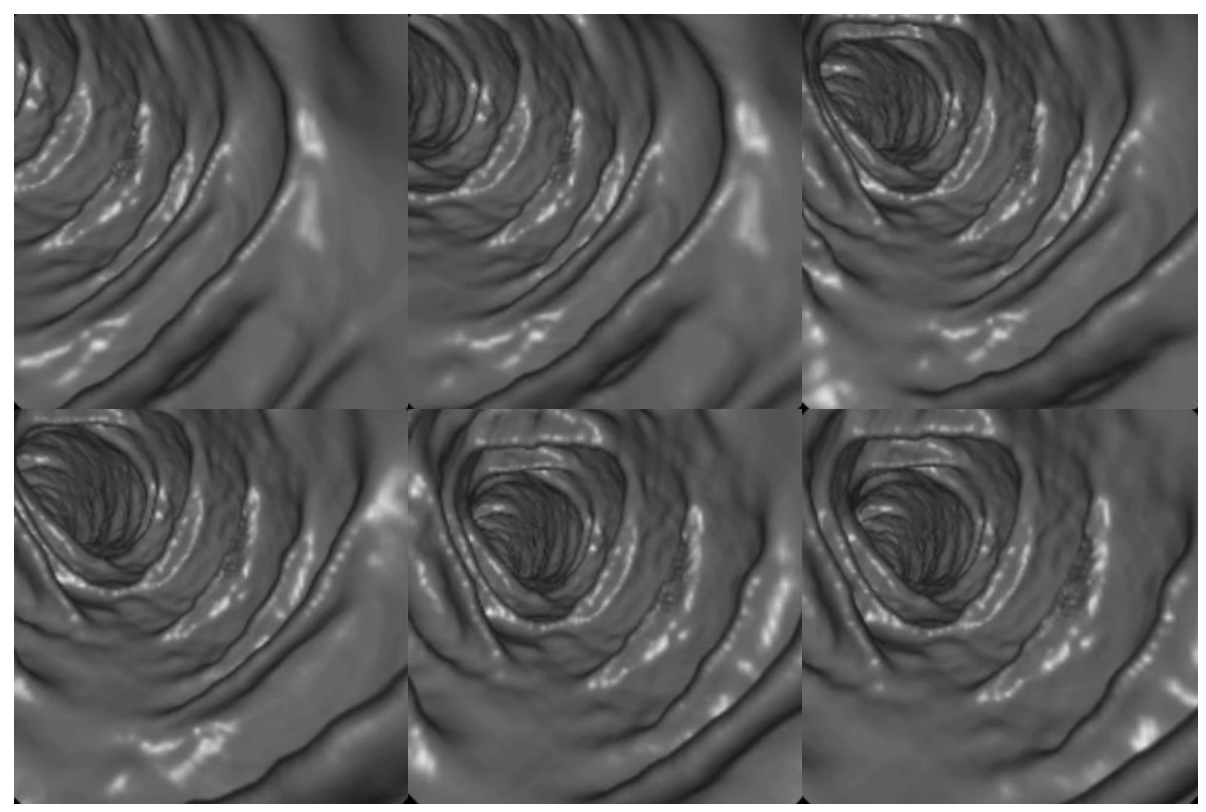

Fig. 7. This is a result of our navigation method. The camera could move forward along the colon cavity automatically

Table 1. comparison of preprocessing time and rendering time of a method that does not perform any kind of collision avoidance (NONE), an object-space guided navigation method using distance map (DIST) and our image space navigation method (OURS) for $512 \times 512 \times 541$ volume data set

\begin{tabular}{cccccc}
\hline & $\begin{array}{c}\text { DIST } \\
(\mathrm{msec})\end{array}$ & $\begin{array}{c}\text { NONE } \\
(\mathrm{msec})\end{array}$ & $\begin{array}{c}\text { OURS } \\
(\mathrm{msec})\end{array}$ & $\begin{array}{c}\text { OURS / } \\
\text { DIST }(\%)\end{array}$ & $\begin{array}{c}\text { OURS / } \\
\text { NONE }(\%)\end{array}$ \\
\hline Preprocessing time & 186880 & - & - & $\mathrm{n} / \mathrm{a}$ & $\mathrm{n} / \mathrm{a}$ \\
Rendering time & 265 & 261 & 278 & $\mathbf{4 . 9 1}$ & $\mathbf{6 . 5 1}$ \\
\hline
\end{tabular}

Object space method doubles up the memory consumption since it should maintain the distance map that has the same size as the original volume data set. On the contrary, our method does not require additional storage. For manipulating $512 \times 512 \times 541$ volume dataset, about 142 Mbytes memory is need for volume data and the sample size storage is required for the distance map. 


\section{Conclusion}

We propose an efficient navigation method that reflects user's control and prohibits a camera from bump against organ wall, when the camera moves through a human cavity such as colon and bronchus. This method makes it possible to perform collision-free navigation and gives us smooth camera movement without trembling. Since it exploits depth information generated in the previous rendering stage, it doesn't require preprocessing step as well as extra storage at all. Using this method, we can go forward and backward anywhere we want to see without consideration about collision between camera and organ wall. Experimental results show us that our method satisfies most of requirement for guided navigation. Future work should be focused on improving this method to obtain more smooth and natural path for camera movement.

\section{References}

1. Levoy, M.: Display of Surfaces from Volume Data, IEEE Computer Graphics and Applications, Vol. 8, No. 3 (1988) 29-37

2. Yagel, R. and Kaufman, A.: Template-based volume viewing, Computer Graphics Forum (Eurographics 92 Proceedings), Cambridge, UK (1992) 153-167

3. Lacroute, P. and Levoy, M.: Fast volume rendering using a shear-wqrp factorization of the viewing transformation, Computer Graphics (SIGGRAPH 94 Proceedings), Orlando, Florida (1994) 451-458

4. Yagel, R. and Shi, Z.: Accelerating volume animation by space-leaping, Proceedings of IEEE Visualization 1993 (1993) 62-69

5. He, T. and Kaufman, A.: Collision detection for volumetric objects, Proceedings of Visualization 1997 (1997)27-34

6. Gibson, S.: Beyond volume rendering: visualization, haptic exploration, and physical modeling of voxel-based objects, $6^{\text {th }}$ Eurographics Workshop on Visualization in Scientific Computing, (1995)

7. De Assis Zampirolli, F. and De Alencar Lotufo, R.: Classification of the distance transformation algorithms under the mathematical morphology approach $\cdot$, Proceedings XIII Brazilian Symposium on Computer Graphics and Image Processing (2000) 292-299

8. Gleicher, M. and Witkin, A.: Through-the lens camera control, ACM SIGGRAPH (1992) 331-340

9. Hong, L., Kaufman, A., Wei, Y., Viswambharan, A., Wax, M. and Liang, Z.: 3D virtual colonoscopy, IEEE Symposium on Biomedical Visualization (1995) 26-32

10. Hong, L., Muraki, S., Kaufman, A., Bartz, D. and He, T.: Virtual voyage: Interactive navigation in the human colon, ACM SIGGRAPH (1997) 27-34 